\title{
An Electronic Circuit System for Time-Reversal of Ultra-Wideband Short Impulses Based on Frequency-Domain Approach
}

\author{
Huiqing Zhai, Shaoshu Sha, Varun K. Shenoy, Student Member, IEEE, Sungyong Jung, Senior Member, IEEE, \\ Mingyu Lu, Senior Member, IEEE, Kyoungwon Min, Sungchul Lee, and Dong S. Ha, Fellow, IEEE
}

\begin{abstract}
In this paper, a compact and low-cost electronic circuit system is designed for time-reversal of ultra-wideband short impulses (with nanosecond and sub-nanosecond temporal durations). A frequency-domain approach is adopted to avoid high sampling rate in time. Specifically, the proposed system obtains the discrete spectra of input impulses first; then realizes time-reversal in frequency domain; and finally synthesizes the time-reversed impulses using discrete continuous wave elements. This system is composed of common and commercially available circuits, and hence, can embody a system-on-chip implementation. Its performance is verified by circuit-electromagnetic co-simulations using impulses with 3-10-GHz frequency band coverage. Advanced Design System and two full-wave Maxwell's equations solvers are used for circuit and electromagnetic simulations, respectively, and their results are coupled and integrated. In the circuit part, most of nonidealities of realistic circuits are taken into account. It is shown by the simulation results that, although realistic circuits unavoidably introduce errors to time-reversal, such errors do not affect the "focusing" phenomena in the context of electromagnetic wave propagation. As a conclusion, the proposed system can be deployed in practical time-reversal communication and radar applications.
\end{abstract}

Index Terms-Electronic circuit system, frequency-domain approach, short impulses, time reversal, ultra-wideband (UWB).

\section{INTRODUCTION}

D UE TO its capability of focusing waves in both space and time, time-reversal technique has attracted enormous interests from engineers, as well as physicists [1]-[3]. Specifically, time-reversal takes advantage of channel reciprocity to achieve "focusing," which typically involves three steps. First, signals propagate through forward channel; second, the signals after passing the forward channel are reversed in time; and finally, the time-reversed signals are transmitted through reverse channel. Channel information is collected through the forward trip. If the forward and reverse channels share the same transfer functions (i.e., reciprocal channel), the channel is compensated during the reverse trip because time-reversal behaves as a space-

Manuscript received March 09, 2009; revised August 28, 2009. First published November 20, 2009; current version published January 13, 2010. This work was supported in part by UTA REP.

H. Zhai, S. Sha, V. K. Shenoy, S. Jung, and M. Lu are with the Department of Electrical Engineering, The University of Texas at Arlington, Arlington, TX 76019 USA (e-mail: huiqing@uta.edu).

K. Min and S. Lee are with the Korea Electronics Technology Institute, Seoul, Korea.

D. S. Ha is with the Electrical and Computer Engineering Department, Virginia Polytechnic Institute and State University, Blacksburg, VA 24061 USA.

Digital Object Identifier 10.1109/TMTT.2009.2035883 time matched filter (time-reversal is equivalent to complex conjugate in frequency domain) [4], [5].

Time-reversal can be applied to both sound waves and electromagnetic waves. In acoustics, time-reversal has been studied extensively [6]-[9]. As a far from complete list, it was successfully implemented for underwater communication [8], [10] and target detection/imaging [11]-[13]. The studies of time-reversal in electromagnetics also have a long history; especially in recent years, a lot of research work on time-reversal for electromagnetic waves has been reported [14]-[24], which is largely driven by the advance of impulse-type ultra-wideband (UWB) technology [25]-[27]. It is demonstrated by many researchers that the combination of time-reversal and UWB makes it possible to develop various novel communication and radar systems [28]-[35] including, but not limited to, multiple-input multiple-output (MIMO) communication [36], indoor communication [37], [38], communications in forest environments [39], buried target detection [24], ground penetrating radar [22], [40], and breast cancer diagnosis [41], [42]. Indeed, the space-time focalization capability associated with time-reversal seems to have unbounded applications: innovative methods and systems based upon time-reversal keep being proposed by researchers in recent years. For instance, time-reversal enables sophisticated "compressed sensing" in radar detection, which allows far fewer measurements than traditional sensing technologies [43]. As another example, since time-reversal can effectively conquer highly dispersive channels, it has potential to realize high-speed wireless communication in complex environments like metallic boxes [44].

Relatively speaking, time-reversal is not so mature a technique in electromagnetics as in acoustics. Although numerous time-reversal UWB communication and radar systems have been proposed, their practical implementations remain challenging tasks. One of the critical difficulties is how to efficiently realize time-reversal of UWB impulses. Compared to sound waves, electromagnetic waves have high operating frequencies (for instance, 3.1-10.6-GHz band was allocated for UWB communication applications by the Federal Communications Commission). As a result, much faster sampling is required to directly sample the impulses and then reverse them. To experimentally investigate time-reversal of electromagnetic waves, some researchers had to resort to expensive high-speed analog-to-digital converters [45]-[52]. As an example, Tektronix TDS6604 digital storage oscilloscope with 20-GSamples/s was used in [46]. In some research efforts, time-reversal 


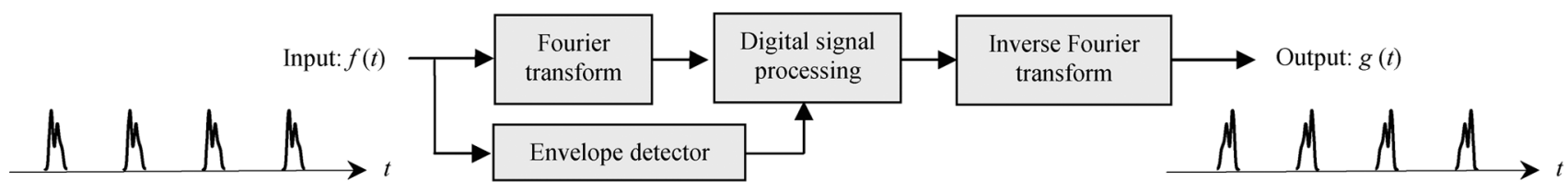

Fig. 1. Block diagram of the proposed time-reversal system.

was realized using vector network analyzers, where data were collected in frequency domain initially and synthesized afterwards [53]-[58]. Microwave signal with $18-\mathrm{GHz}$ bandwidth was time reversed in [59] with aids of up-conversion to optical carrier and nonlinear optical complex conjugate. Obviously, to accomplish practical time-reversal communication and radar systems, efficient and low-cost techniques to reverse UWB impulses are called for. In this paper, an electronic circuit system is designed to time reverse UWB impulses (with nanosecond and sub-nanosecond temporal durations). A frequency-domain approach is adopted to avoid high sampling rate in the time domain. Specifically, time-reversal is achieved in the following three stages.

1) Fourier transform, to obtain discrete spectra of input impulses.

2) Digital signal processing in frequency domain, to carry out complex conjugate of the spectra obtained in 1).

3) Inverse Fourier transform, to synthesize reversed impulses based on the processed spectra in 2).

The proposed system is composed of common semiconductor electronic circuits, including oscillators, multipliers/mixers, bandpass filters (BPFs), amplifiers, phase shifters, and switches. Thus, it is compact and low cost; moreover, it can embody a system-on-chip implementation. It is worth noting that, the authors are not the first ones who attempt applying frequency-domain approaches to UWB signal generation and reception. Sub-band analysis [60], [61] and bank of passive resonators [62] were investigated before to relieve the high sampling rate problem in UWB receivers; and, Fourier synthesis was suggested for UWB signal generators as early as in 1997 [63]. However, to the best of the authors' knowledge, the hardware architecture in this paper, which combines Fourier transform and inverse Fourier transform circuits to accomplish time-reversal of UWB signals, had never been reported. Basic concept of the system proposed in this paper was presented by the authors in [64]; here, complete description and verification are provided. The proposed circuit system is simulated by the Advanced Design System (ADS) with commercially available components; most of the nonidealities of realistic circuits are taken into account. Simulation results using impulses with around 1-ns temporal width and [3], [10] gigahertz spectral coverage demonstrate real-time and reliable time-reversal, even with the presence of strong noise. Moreover, performance of the proposed time-reversal circuit system is verified in the context of electromagnetic propagation. Circuit-electromagnetic co-simulations are carried out for two real-world problems (one is for wireless communication and the other is for radar detection). Two full-wave solvers, finite difference time domain (FDTD) [65] and method of moments (MoM) [66], are used to solve the Maxwell's equations; and they are coupled with the circuit simulator. Electromagnetic propagation results with ideal time-reversal and practical time-reversal (i.e., time-reversal using circuits in this paper) are compared. In both cases, desired "focusing" phenomena are observed. It is, therefore, concluded that, errors introduced by the time-reversal circuits are tolerable and the proposed system can be deployed in practical communication and radar applications.

This paper is organized as follows. In Section II, the proposed time-reversal circuit system is described in detail. Section III presents results from both circuit simulation and circuit-electromagnetic co-simulation, to demonstrate the performance of the system in Section II. Finally, conclusions of this study are drawn in Section IV.

\section{Description of the Proposed Time-Reversal System}

The proposed time-reversal circuit system is described in this section. Section II-A presents the system design. Three major blocks of the system, which include Fourier transform, inverse Fourier transform, and digital signal processing, are discussed in Sections II-B-D, respectively.

\section{A. System Design}

Block diagram of the proposed time-reversal system is depicted in Fig. 1. The input is a periodic signal consisting of a series of short impulses (with nanosecond or sub-nanosecond temporal durations). The time-reversal system has three major blocks.

1) Fourier transform block obtains discrete spectrum of the input signal.

2) Digital signal processing block processes the spectrum obtained in 1).

3) Inverse Fourier transform block makes use of spectrum in 2) to synthesize the output, which is a periodic signal with each impulse in the input signal reversed.

In addition to the three major blocks above, envelope detector block acquires the positions of impulses in the input signal to facilitate signal processing.

It is assumed that the input signal $f(t)$ has period $T_{0}$ and there is a short impulse within each period, as illustrated in Fig. 2. The short impulse within interval $t \in\left[0, T_{0}\right]$ has starting time $t_{a}$ and ending time $t_{b}$. Since $f(t)$ is periodic, it can be represented by Fourier series as

$$
f(t)=\sum_{m=-\infty}^{+\infty} c_{m} e^{j m \omega_{0} t}
$$

where $j=\sqrt{-1}, \omega_{0}=2 \pi / T_{0}$, and

$$
c_{m}=\frac{1}{T_{0}} \int_{0}^{T_{0}} f(t) e^{-j m \omega_{0} t} d t .
$$

Also, it is assumed that the input signal's discrete spectrum is virtually limited within minimum frequency $\omega_{\min }$ and max- 


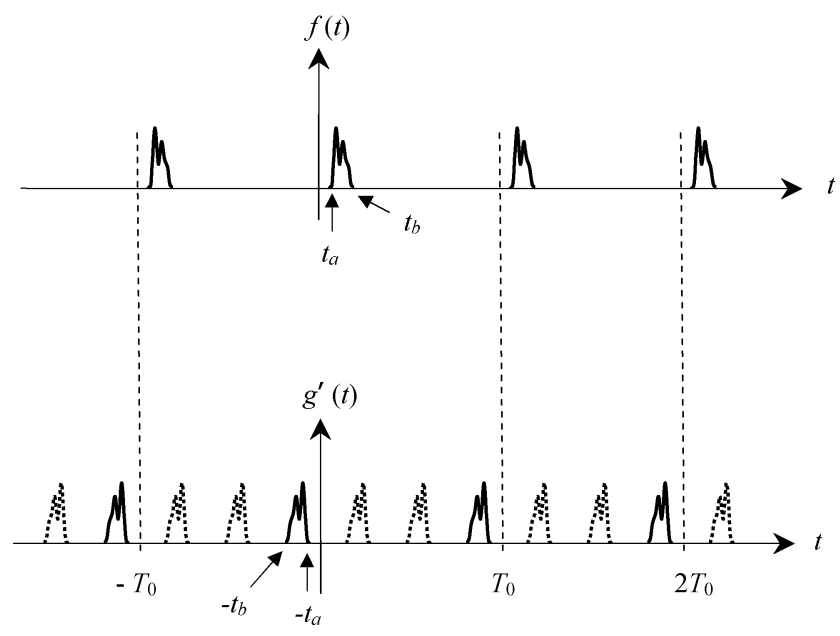

Fig. 2. Illustration of signals in the proposed time-reversal system.

imum frequency $\omega_{\max }$. That is, $\left|c_{m}\right| \approx 0$ when $\left|m \omega_{0}\right|>\omega_{\max }$ or $\left|m \omega_{0}\right|<\omega_{\min }$ (Fig. 2).

In the Fourier transform block of the proposed system, a subset of $\left\{c_{m}\right\}$ is selected. The elements of this subset are denoted $\left\{\tilde{A}_{1}, \tilde{A}_{2}, \ldots, \tilde{A}_{N}\right\}$, and they are Fourier series of $f(t)$ located at $\left\{\omega_{1}, \omega_{2}, \ldots, \omega_{N}\right\}$, where $\omega_{n}=\omega_{1}+(n-1) \chi \omega_{0}, n=$ $1,2, \ldots, N$

$$
N=\left\lceil\frac{\omega_{\max }-\omega_{\min }}{\chi \omega_{0}}\right\rceil+1
$$

$\chi \geq 1$ is termed the undersampling ratio and is chosen as

$$
\chi=\left\lfloor\frac{2 \pi}{\omega_{0}\left(t_{b}-t_{a}\right)}\right\rfloor
$$

and the two operators $\lfloor\cdot\rfloor$ and $\lceil\cdot\rceil$ find the nearest integers smaller and greater than arguments respectively. Next, complex conjugate is carried out onto the selected spectral samples $\left\{\tilde{A}_{n}\right\}$, in the digital signal processing block. Finally in the inverse Fourier transform block, the processed spectral samples are used to construct $g^{\prime}(t)$

$$
g^{\prime}(t)=2 \operatorname{Re}\left\{\sum_{n=1}^{N} \overline{\tilde{A}_{n}} e^{j \omega_{n} t}\right\}
$$

where $\tilde{A}_{n}$ is the complex conjugate of $\tilde{A}_{n}$. Due to undersampling, temporal period of $g^{\prime}(t)$ is $T_{0} / \chi$. Desired output $g(t)$ can be obtained from $g^{\prime}(t)$ by removing unnecessary impulses. (In Fig. 2, $g^{\prime}(t)$ is illustrated with $\chi=3$ and unnecessary impulses are denoted by dashed lines.) The envelope detector block assists determining which impulses to remove.

Detailed block diagram of the proposed system is given in Fig. 3. Among the four blocks, envelope detector block is the simplest. It follows a standard amplitude de-modulator design, where $\omega_{c}=\left(\omega_{\min }+\omega_{\max }\right) / 2$. The output of low-pass-filter in the envelope detector block of Fig. 3 contains narrow impulses and the rough locations of these impulses are measured to be $t_{k}^{*}$ and $t_{k}^{*} \in\left[k T_{0}+t_{a}, k T_{0}+t_{b}\right]$ for $k=\ldots,-1,0,1,2, \ldots$. The other three blocks, Fourier transform, inverse Fourier transform, and digital signal processing, are discussed in Sections II-B-D, respectively.
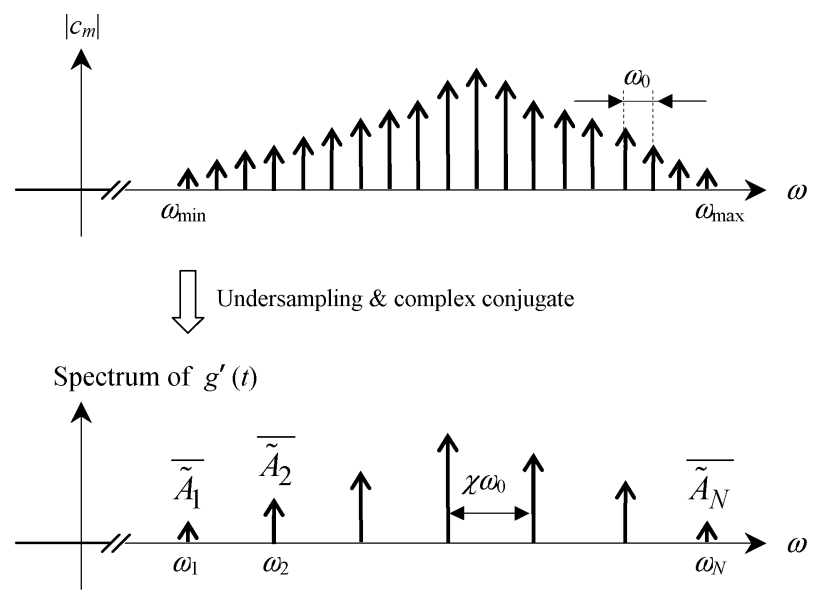

\section{B. Fourier Transform Block}

The Fourier transform block makes use of $N$ local oscillators to find $N$ spectral samples of the input signal. Specifically, the frequencies of local oscillators are $\omega_{n}-\omega_{0} / 4, n=1,2, \ldots, N$. The mixers followed by oscillators behave as down-converters. After down-conversion by the $n$th oscillator, the spectrum of the input signal is depicted in Fig. 4. Note that $f(t)$ 's spectral sample at $\omega_{n}$ is moved to location $\omega=\omega_{0} / 4$ after down-conversion; and its sample at $-\omega_{n}$ is relocated to $\omega=-\omega_{0} / 4$. BPFs centered at frequency $\omega_{0} / 4$ and with sufficiently small bandwidth filter out spectral sample $\tilde{A}_{n}$. In Fig. 4, the BPF's frequency response is denoted by two impulses with dashed lines. Since the output of BPF is a low-frequency signal (i.e., oscillating at frequency $\omega_{0} / 4$ ), its magnitude and phase can be easily detected and are denoted as complex phasor $A_{n}$. Apparently

$$
A_{n}=\tilde{A}_{n} e^{-j \phi_{n}}
$$

where $\phi_{n}$ is the phase of the $n$th local oscillator. The above architecture is superheterodyne, where an intermediate frequency $\left(\omega_{0} / 4\right)$ is processed after down-conversion. It is noted that superheterodyne processing is not the only option to analyze the input signal's spectrum. In [62], passive resonators are used to catch the spectral lines directly. In [64], analog correlator configuration ("multiplier + integrator") was exploited to realize the Fourier transform. The superheterodyne architecture is adopted here, because it offers two unique advantages. First, down-conversion avoids processing the input signal at high frequencies; in contrast, resonators with high quality factors must be built at gigahertz range with accurate resonant frequencies in [62]. Second, processing at intermediate frequency is much easier than at dc; for instance, the analog correlator design in [64] is sensitive to the isolation ratio in mixers while this issue is suppressed by the BPFs in the scheme in Fig. 3 .

A few accessory circuits are needed to secure desirable operation of the Fourier transform block; and they are explained in the remainder of this section.

Frequencies of the $N$ local oscillators must be locked to preset values. Instead of regular phase-locked loops, a simple locking circuit is proposed for the system in this paper. As 


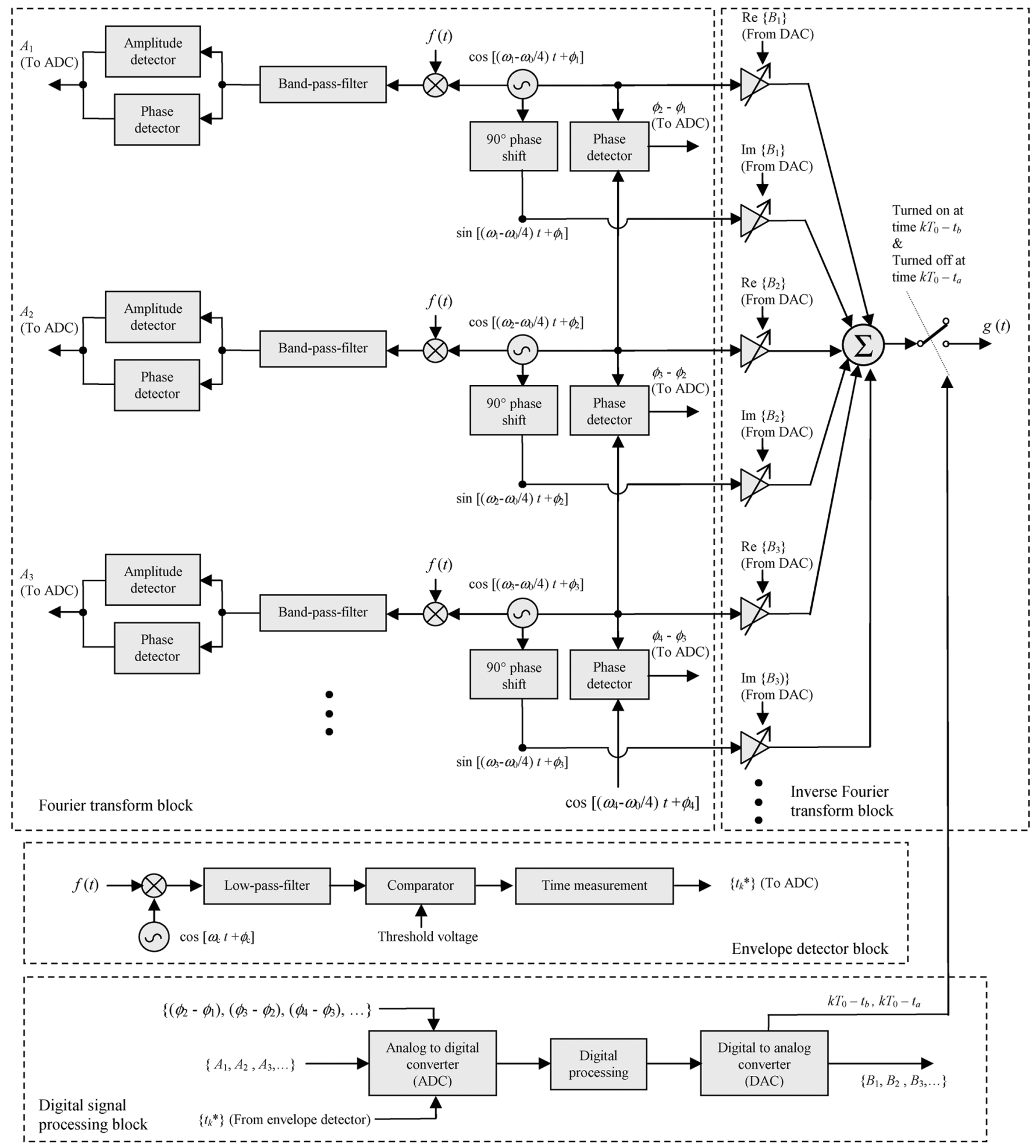

Fig. 3. Detailed schematic diagram of the proposed time-reversal system.

shown in Fig. 5, the $n$th oscillator's frequency is controlled by a bias signal as $\omega_{n}-\omega_{0} / 4+\Delta \omega$. This oscillator is mixed with the input signal $f(t)$; and the mixer's output goes to a BPF centered at $\omega_{0} / 4$ and with a high quality factor (high $Q$ ). Since the input signal is periodic, the output of BPF is maximized when $\Delta \omega=0$. Obviously, the narrower the BPF's bandwidth is, the smaller phase error there is. However, if the BPF has too high a quality factor, a lot of time is needed for it to reach steady state, and consequently, the locking speed is low. In practice, a medium bandwidth should be chosen such that BPFs in Fig. 3 could capture desired spectral lines with sufficient accuracy.

Phase of the first local oscillator, $\phi_{1}$, is assumed to be zero; and phases of the other oscillators are obtained through phase detectors in Fig. 3. The block diagram of phase detector be- 


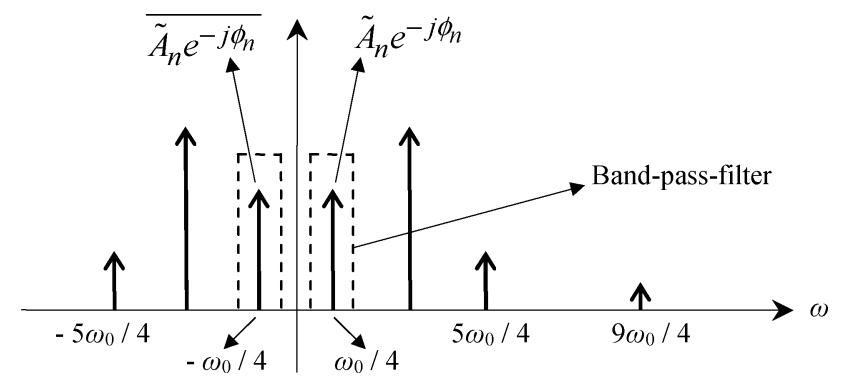

Fig. 4. Illustration of spectrum after down-conversion with the $n$th oscillator.

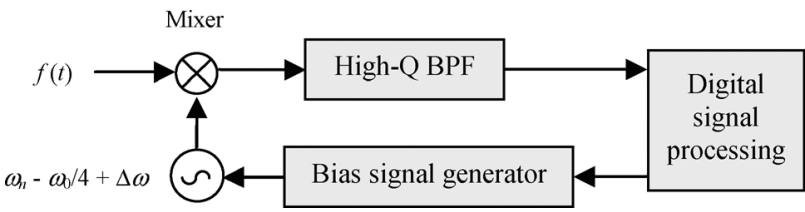

Fig. 5. Block diagram of the frequency locking circuit.

tween oscillators at $\omega_{n-1}-\omega_{0} / 4$ and $\omega_{n}-\omega_{0} / 4$ is shown in Fig. 6. The two continuous-wave signals are multiplied and only the difference frequency component survives after the BPF. It is noted that, the output after BPF is oscillating at frequency $\omega_{n}-\omega_{n-1}=\chi \omega_{0}$. When this signal is compared with an appropriately chosen threshold voltage, periodic pulses are resulted, as shown in Fig. 6. Apparently, the edges' timings in the comparator's output are directly related to phase difference $\phi_{n}-\phi_{n-1}$

$$
\phi_{n}-\phi_{n-1}=-\phi_{\mathrm{BPF}}-\frac{t_{1}+t_{2}}{2}\left(\omega_{n}-\omega_{n-1}\right)
$$

where $\phi_{\mathrm{BPF}}$ is the phase response of the BPF in Fig. 6 at frequency $\omega_{n}-\omega_{n-1}$. Composition of the time measurement block in Fig. 6 is plotted in Fig. 7. The rise/fall edge samples a ramp signal and a digital counter. The ramp signal has period $T_{0} / \chi$. When the ramp signal is sampled, its output voltage is linearly proportional to a time between 0 and $T_{0} / \chi$. At the same time, the clock shares the same period $T_{0} / \chi$ and the counter counts the number of $T_{0} / \chi$ that have passed. Both the ramp signal and clock are reset every $T_{0}$. Consequently, the samplings of ramp signal and digital counter jointly measure the timing in the range of $\left[0, T_{0}\right]$.

In addition, isolators/buffers must be put between the input signal $f(t)$ and every mixer to avoid couplings among the multiple local oscillators.

\section{Inverse Fourier Transform Block}

The inverse Fourier transform block exploits the same set of oscillators as those in the Fourier transform block. The adder circuit in Fig. 8 combines the $N$ oscillators' outputs. Magnitudes and phases of the $N$ continuous-wave elements are adjusted by $2 N$ variable gain amplifiers (Fig. 3). As a result, the synthesized signal after adder is

$$
g^{\prime}(t)=\frac{R_{F}}{R} \operatorname{Re}\left\{\sum_{n=1}^{N} B_{n} e^{j\left(\left(\omega_{n}-\frac{\omega_{0}}{4}\right) t+\phi_{n}\right)}\right\}
$$

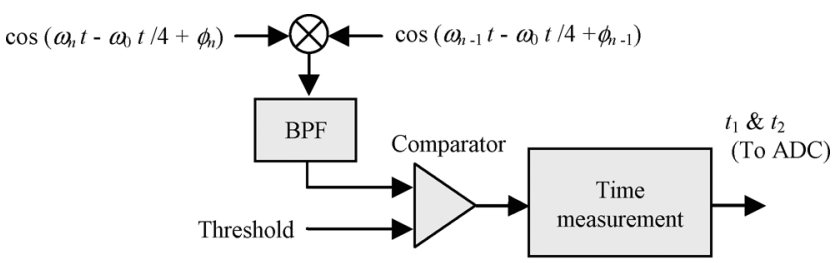

(a)

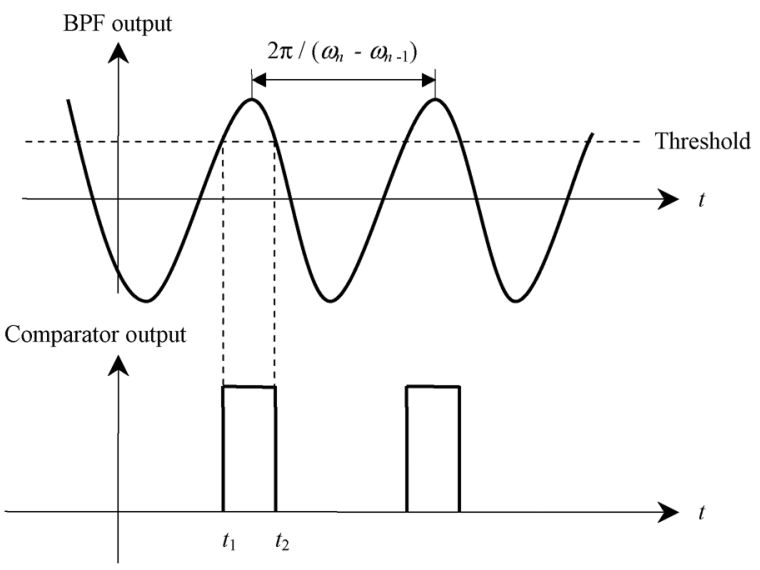

(b)

Fig. 6. Block diagram of phase detector circuit. (a) Circuit block diagram. (b) Illustrations of outputs of BPF and comparator.

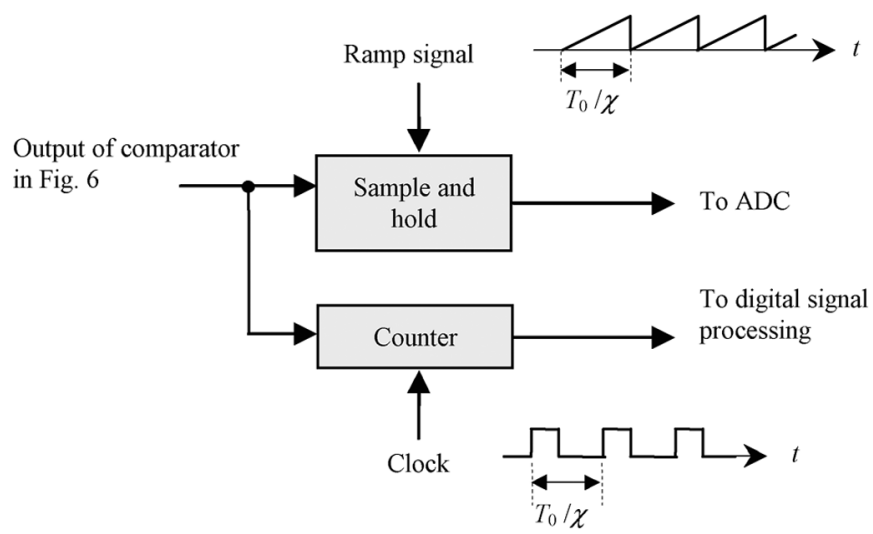

Fig. 7. Block diagram of time measurement circuit.

where $R_{F}$ and $R$ are the two resistances in Fig. 8. The desired output $g(t)$ is obtained by multiplying $g^{\prime}$ by windowing function $W(t)$

$$
g(t)=g^{\prime}(t) W(t)
$$

and

$W(t)$

$= \begin{cases}1, & t \in\left[k T_{0}-t_{b}, k T_{0}-t_{a}\right], \quad k=\ldots,-1,0,1,2, \ldots \\ 0, & \text { elsewhere }\end{cases}$

Physically, multiplication with the windowing function is realized by a switch after the adder. 


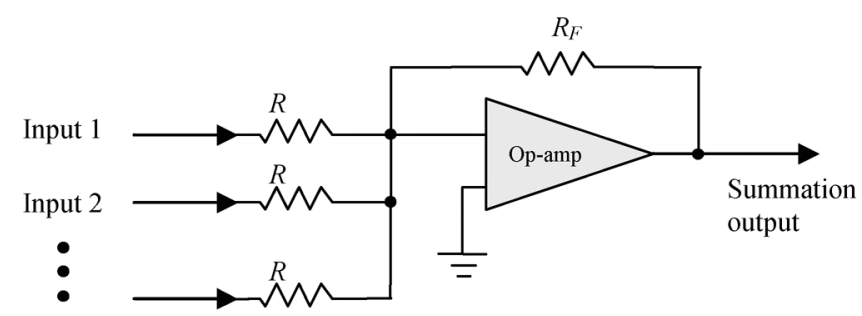

Fig. 8. Block diagram of adder in inverse Fourier transform block.

\section{Digital Signal Processing Block}

The digital signal processing block has two major functionalities, which are: 1) to obtain coefficients $\left\{B_{n}\right\}$ and 2) to find switching timings $k T_{0}-t_{b}$ and $k T_{0}-t_{a}, k=$ $\ldots,-1,0,1,2, \ldots$.

Comparison among (5), (6), and (8) yields that, for the inverse Fourier transform block to generate desired $g^{\prime}(t)$ through (8), coefficients $\left\{B_{n}\right\}$ should be chosen as

$$
B_{n}=\overline{A_{n}} e^{-j 2 \phi_{n}} e^{j \frac{\omega_{0}}{4}\left[(2 k+1) T_{0}-t_{k}^{*}\right]}
$$

when

$$
t \in\left[k T_{0},(k+1) T_{0}\right]
$$

Equation (11) relies on the fact that $W(t)$ is a very narrow window around time $(2 k+1) T_{0}-t_{k}^{*}$; hence, $\exp \left\{j \omega_{0} t / 4\right\} \cong$ $\exp \left\{j \omega_{0}\left[(2 k+1) T_{0}-t_{k}^{*}\right] / 4\right\}$ when $t$ is close to $(2 k+1) T_{0}-t_{k}^{*}$.

To find $t_{a}$ and $t_{b}$, time-domain signal $\hat{f}(t)$ is reconstructed in the digital signal processing block using $\left\{A_{n}\right\}$ obtained by the Fourier transform block

$$
\begin{aligned}
\hat{f}(t) & =2 \operatorname{Re}\left\{\sum_{n=1}^{N} A_{n} e^{j\left(\omega_{n} t+\phi_{n}\right)}\right\} \\
& =2 \operatorname{Re}\left\{\sum_{n=1}^{N} \tilde{A}_{n} e^{j \omega_{n} t}\right\} .
\end{aligned}
$$

Obviously, signal $\hat{f}(t)$ is the "undersampled version" of input signal $f(t)$. It has period $T_{0} / \chi$. In other words, there are $\chi$ impulses within one $T_{0}$ (the period of the input signal). The relationship between $f(t)$ and $\hat{f}(t)$ is illustrated in Fig. 9, when $\chi=3$. To identify which one of the $\chi$ impulses coincides with the input signal, the following Fourier transform is performed:

$$
\begin{gathered}
\hat{F}_{m}=\frac{\chi}{T_{0}} \int_{t_{0}}^{t_{0}+T_{0} / \chi} \hat{f}(t) e^{-j m \omega_{0} t} d t, \\
m=\ldots,-1,0,1,2, \ldots
\end{gathered}
$$

In the above, $t_{0} \in\left[t_{0}^{*}-T_{0} / \chi, t_{0}^{*}\right]$ is a variable, and $t_{0}^{*}$ is the rough location of the impulse of $f(t)$ in the range $t \in\left[0, T_{0}\right]$. The value of $t_{0}$ that minimizes the relative error between $\left\{\left|\hat{F}_{m}\right|\right\}$ and

\begin{tabular}{|c|c|c|c|}
\hline Component & Company & Model & Parameters \\
\hline \multirow{3}{*}{ Op-amp } & \multirow{3}{*}{$\begin{array}{c}\text { Texas } \\
\text { Instruments }\end{array}$} & \multirow{3}{*}{ THS4302 } & Gain-bandwidth product: $12 \mathrm{GHz}$ \\
\hline & & & Slew rate: $5500 \mathrm{~V} / \mathrm{us}$ \\
\hline & & & Gain: $14 \mathrm{~dB}$ \\
\hline \multirow{4}{*}{$\begin{array}{c}\text { Local } \\
\text { oscillator }\end{array}$} & \multirow{4}{*}{ Hittite } & \multirow{4}{*}{$\begin{array}{c}\text { HMC587LC4B } \\
\text { HMC388LP4 } \\
\text { HMC506LP4 }\end{array}$} & Frequency range: $3.15-10 \mathrm{GHz}$ \\
\hline & & & Power output: $5 \mathrm{dBm}$ \\
\hline & & & Phase noise: -95 dBc/Hz@100 kHz \\
\hline & & & 2nd harmonic: $-15 \mathrm{dBc}$ \\
\hline \multirow{5}{*}{ Mixer } & \multirow{5}{*}{$\begin{array}{l}\text { Teledyne } \\
\text { Cougar }\end{array}$} & \multirow{5}{*}{ MMP12241 } & Bandwidth: $2-13 \mathrm{GHz}$ \\
\hline & & & IP3: $10 \mathrm{dBm}$ \\
\hline & & & Conversion loss: $5 \mathrm{~dB}$ \\
\hline & & & LO-RF isolation ratio: $35 \mathrm{~dB}$ \\
\hline & & & Noise figure: $8.5 \mathrm{~dB}$ \\
\hline \multirow{4}{*}{$\begin{array}{c}\text { Band-pass } \\
\text {-filter }\end{array}$} & \multirow{4}{*}{$\mathrm{AMCrf}$} & \multirow{4}{*}{ AM21.4CR167 } & Center frequency: $21.4 \mathrm{MHz}$ \\
\hline & & & Bandwidth: $\pm 10 \mathrm{KHz}$ \\
\hline & & & Insertion loss: $2.5 \mathrm{~dB}$ \\
\hline & & & Pass-band ripple: $1 \mathrm{~dB}$ \\
\hline \multirow{3}{*}{$\begin{array}{c}\text { Variable } \\
\text { gain } \\
\text { amplifier }\end{array}$} & \multirow{3}{*}{ Hittite } & \multirow{3}{*}{$\begin{array}{l}\text { HMC625LP5 } \\
\text { HMC694LP4 }\end{array}$} & Bandwidth: DC $-17 \mathrm{GHz}$ \\
\hline & & & Tuning range of gain: $31.5 \mathrm{~dB}$ \\
\hline & & & Gain flatness: $\pm 1 \mathrm{~dB}$ \\
\hline \multirow{4}{*}{ Phase shifter } & \multirow{4}{*}{ Harley } & \multirow{4}{*}{$\begin{array}{l}7724 \mathrm{~A} \\
7722 \mathrm{~A}\end{array}$} & Frequency range: $2-12 \mathrm{GHz}$ \\
\hline & & & Control type: 10-bit digital \\
\hline & & & Resolution: 1.41 degrees \\
\hline & & & 2nd harmonic: $-30 \mathrm{dBc}$ \\
\hline
\end{tabular}
$\left\{\left|c_{m}\right|\right\}$ is picked as $t_{a}$, and $t_{b}=t_{a}+T_{0} / \chi$. In this paper, $\left\{\left|c_{m}\right|\right\}$

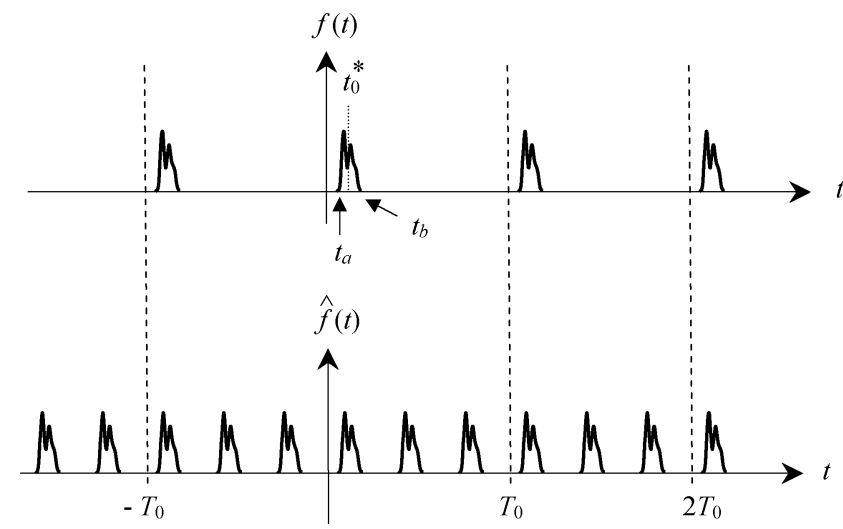

Fig. 9. Illustration of algorithm to find $t_{a}$ and $t_{b}$.

TABLE I

PARAMETERS OF KEY COMPONENTS USED IN ADS SIMULATION

are obtained by linear interpolation among $\left\{\left|A_{n}\right|\right\}$, which are results of the Fourier transform block.

\section{Simulation Results}

In this section, the time-reversal circuit system proposed in Section II is verified by circuit simulation and circuit-electromagnetic co-simulation. Results from circuit simulation and circuit-electromagnetic co-simulation are presented in Sections III-A and B, respectively.

\section{A. Circuit Simulation Results}

The system in Fig. 3 is simulated by ADS. Commercial products with practical parameters are used for all the major components including local oscillators, mixers, variable gain amplifiers, phase shifters, BPFs, and op-amps. Model numbers and key parameters of these components are listed in Table I. Most of the nonidealities of realistic circuits are taken into account. For instance, the mixers have 35-dB isolation in between the two input ports; the variable gain amplifiers have gain range about $30 \mathrm{~dB}$ and phase response fluctuation $\pm 30^{\circ}$; and gain-bandwidth product of the op-amp is $12 \mathrm{GHz}$. 


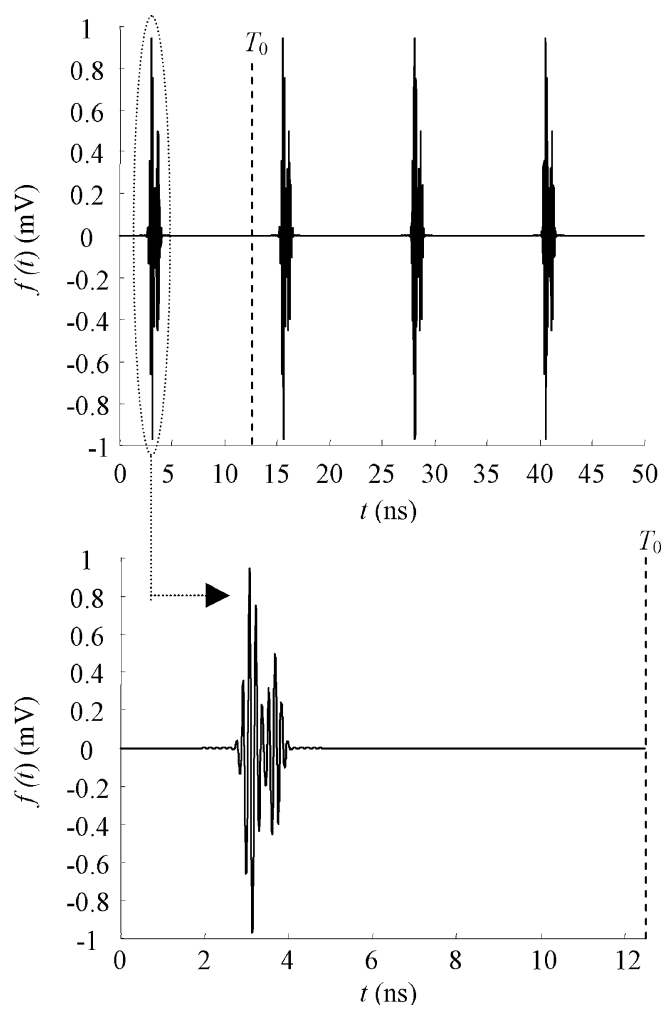

Fig. 10. Input signal to the time-reversal circuit (without noise).
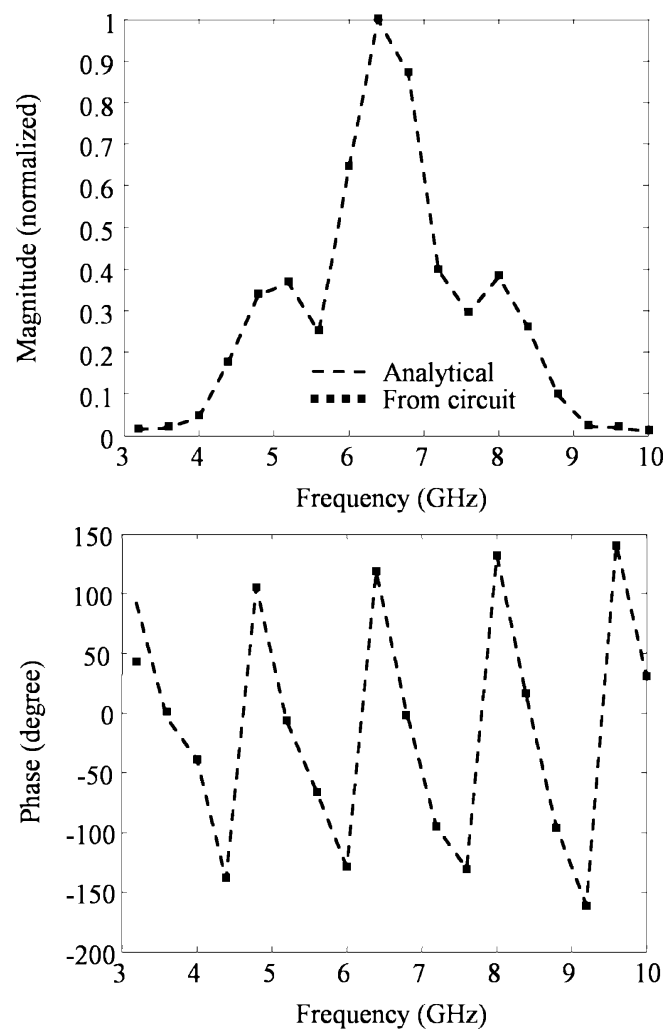

Fig. 11. Output of Fourier transform block (without noise).

In the simulation results of this section, the input signal to the time-reversal system is periodic with $T_{0}=12.5 \mathrm{~ns}$ and

$$
f(t)=\left[e^{-\frac{\left(t-t_{\alpha}\right)^{2}}{2 \sigma^{2}}}+\frac{1}{2} e^{-\frac{\left(t-t_{\beta}\right)^{2}}{2 \sigma^{2}}}\right] \cos \left(\omega_{c} t\right), \quad t \in\left[0, T_{0}\right]
$$
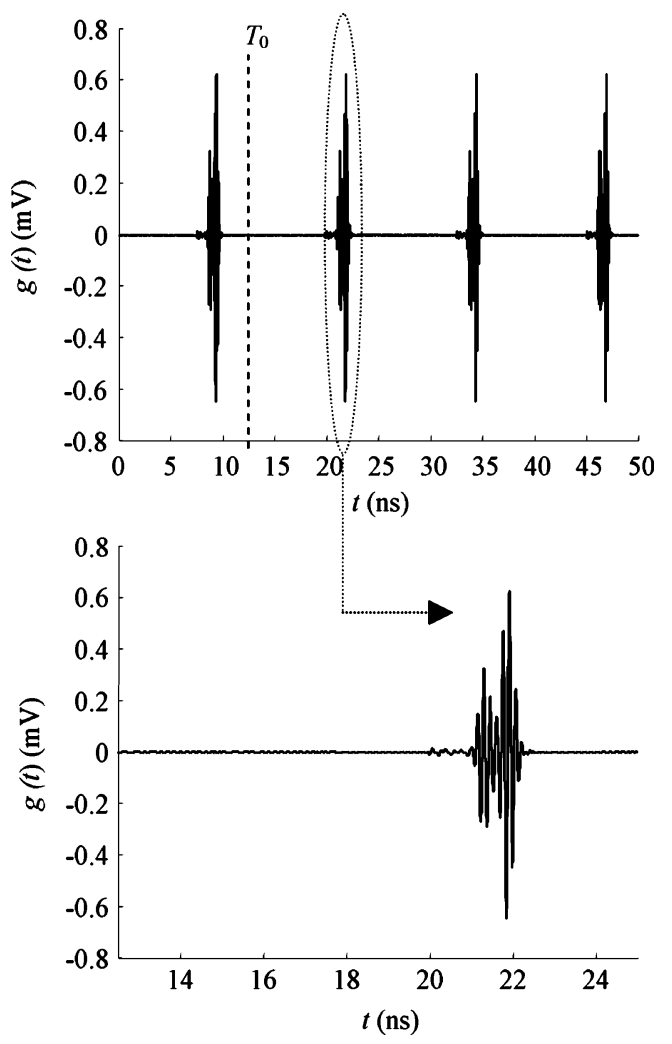

Fig. 12. Output signal of the time-reversal circuit (without noise).

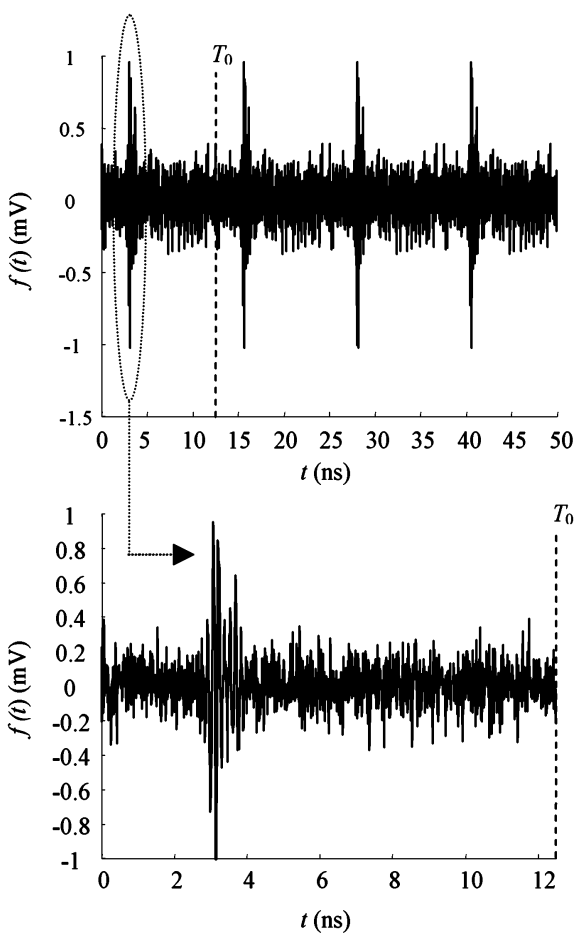

Fig. 13. Input signal to the time-reversal circuit (with noise).

where $\sigma=138 \times 10^{-12} \mathrm{~s}, t_{\alpha}=3.125 \times 10^{-9} \mathrm{~s}, t_{\beta}=t_{\alpha}+4 \sigma$, and $\omega_{c}=2 \pi \times 6.5 \times 10^{9} \mathrm{rad} / \mathrm{s}$. Input signal in (14) is plotted in Fig. 10: it consists of two modulated Gaussian pulses, with total temporal duration about $1.4 \mathrm{~ns}$ and spectral coverage approximately from 3 to $10 \mathrm{GHz}$. In all the results in this section, 

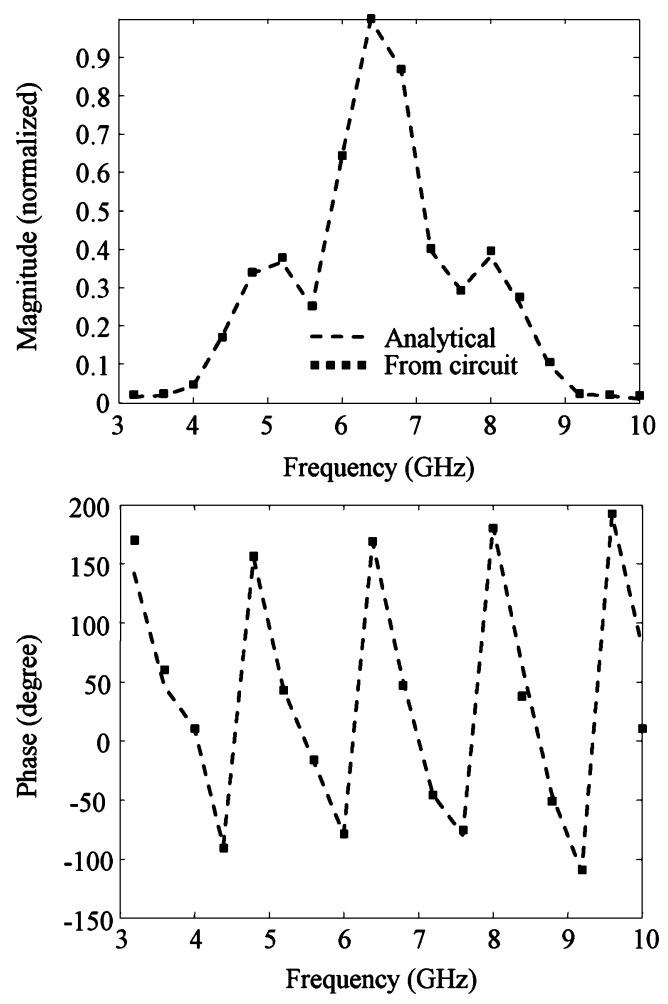

Fig. 14. Output of Fourier transform block (with noise).

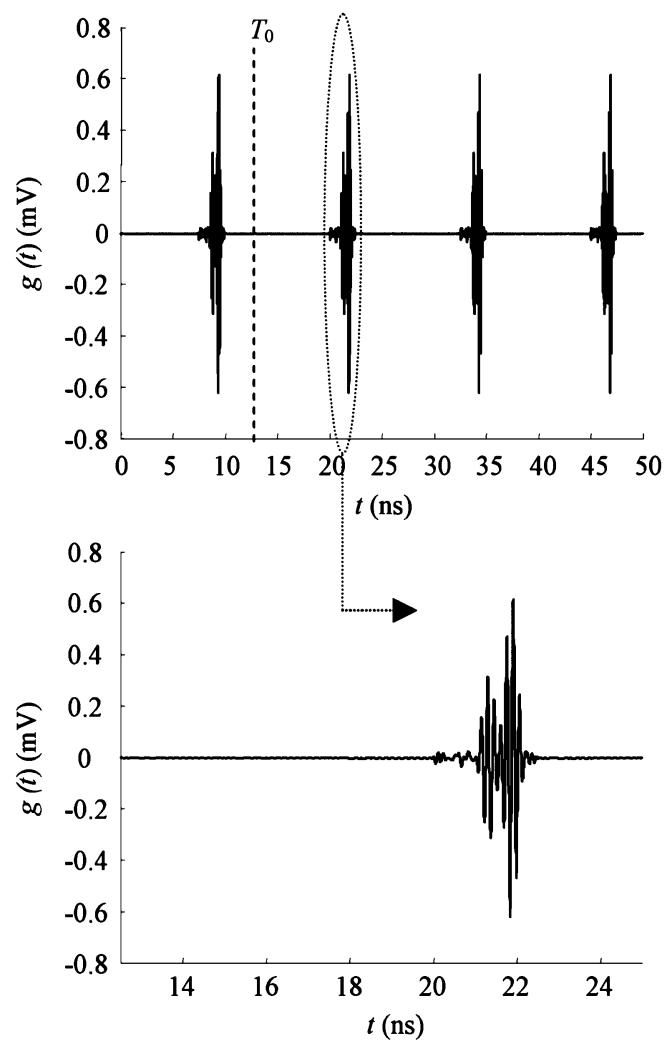

Fig. 15. Output signal of the time-reversal circuit (with noise).

$\chi=5, N=18$, and $\omega_{1}=2 \pi \times 3.2 \times 10^{9} \mathrm{rad} / \mathrm{s}$. The output of Fourier transform block is shown in Fig. 11 and has excellent agreement with analytical spectrum of (14). The waveform

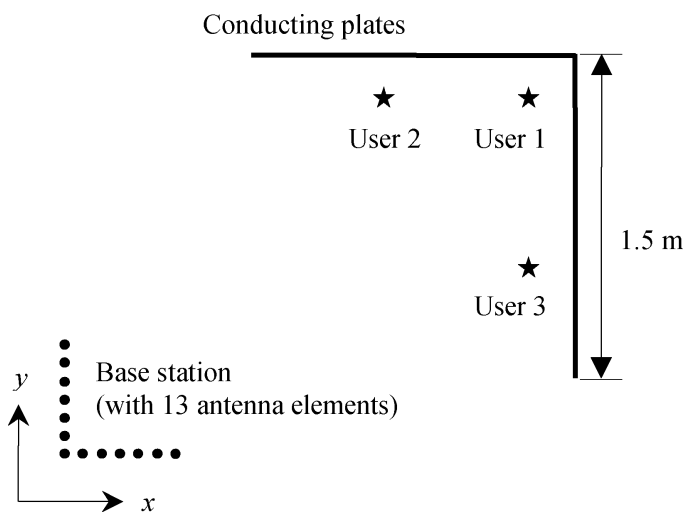

Fig. 16. Geometry of the wireless communication example.

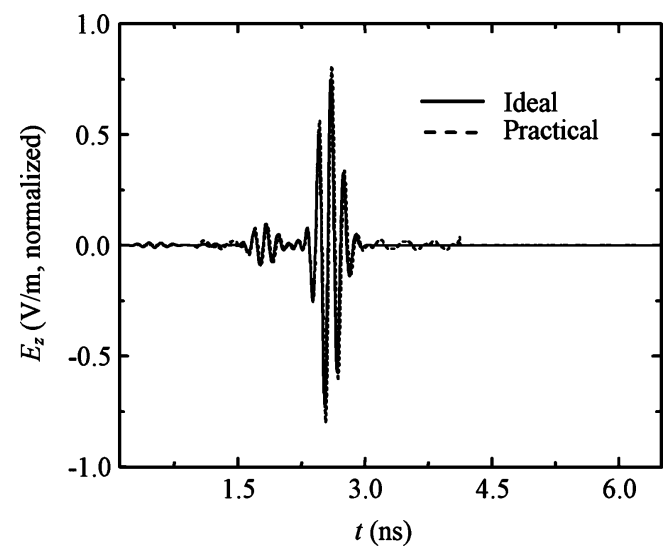

Fig. 17. Comparison of ideal and practical time-reversal at one of the antenna elements in the communication example.

after inverse Fourier transform block, $g(t)$, is plotted in Fig. 12. It is observed that, the proposed system successfully achieves time-reversal of the input signal. Relative to the Fourier transform block, the inverse Fourier transform block has more error sources, most of which are from the phase fluctuations of variable gain amplifiers and adder. From our experience, when the phase fluctuation is within $\pm 40^{\circ}$ degrees range, the ultimate time-reversed waveforms are usually acceptable.

The proposed time-reversal system is also tested in noisy scenarios. In Fig. 13, the input signal is plotted when a pretty strong additive white Gaussian noise is added to it. The signal-to-noise ratio is about $0 \mathrm{~dB}$. When the signal in Fig. 13 is input into the system, outputs of Fourier transform block and inverse Fourier transform block are shown in Figs. 14 and 15, respectively. With the presence of strong noise, the proposed system reliably fulfills the time-reversal job. As a matter of fact, since the input signal is periodic in time and noise has continuous spectrum, signal-to-noise ratio after the Fourier transform block is much better than $0 \mathrm{~dB}$. To a large extent, it is due to the narrow bandwidth of BPFs in Fig. 3, which is chosen as $300 \mathrm{kHz}$ in this section.

\section{B. Circuit-Electromagnetic Co-Simulation Results}

In this section, the proposed time-reversal system is evaluated in the context of electromagnetic wave propagation. Realistic 


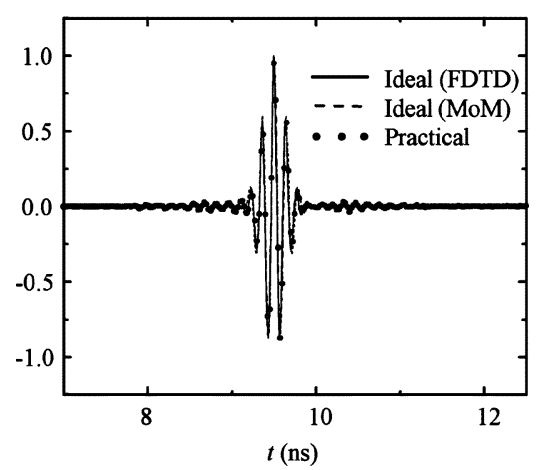

(a)

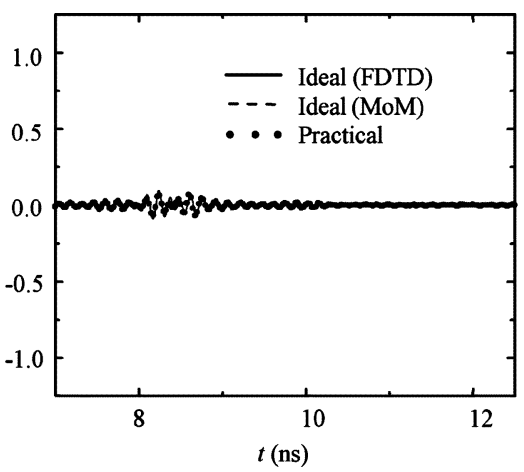

(b)

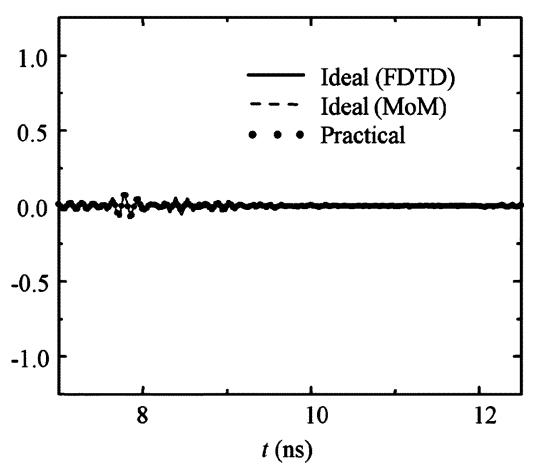

(c)

Fig. 18. Signals received at the three users when there is a link between the base station and User 1. (The vertical axes are normalized $E_{z}$.) (a) At User 1. (b) At User 2. (c) At User 3.

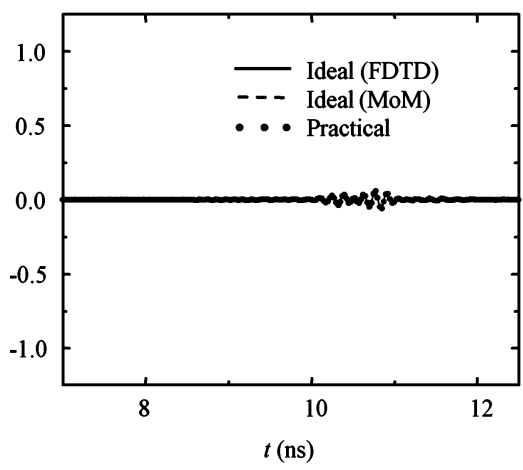

(a)

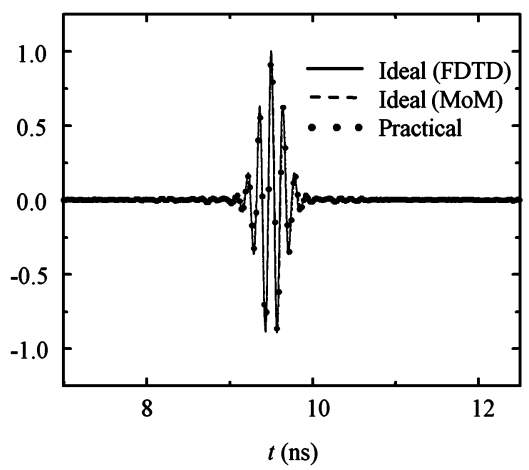

(b)

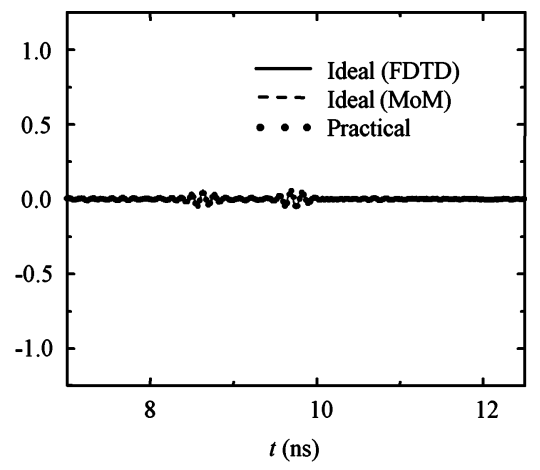

(c)

Fig. 19. Signals received at the three users when there is a link between the base station and User 2. (The vertical axes are normalized $E_{z}$.) (a) At User 1. (b) At User 2. (c) At User 3.

time-reversal communication and radar problem configurations are studied, which involves wave propagation, signal reception, and time-reversal of signals. The electromagnetic waves' propagation and reception are simulated by two Maxwell's equations solvers: one is based on the FDTD method [65], and the other on the MoM [66]. The FDTD solver obtains solutions in time domain; while the MoM solver is carried out in frequency domain and its results are inverse Fourier transformed to time domain afterwards. It is noted that both the FDTD and MoM simulators are full-wave solvers: they incorporate all the electromagnetic coupling and interaction phenomena. In this section, the electromagnetic solvers are always executed for the following two cases.

1) Ideal time-reversal. In this case, the FDTD and MoM solvers are independent of the circuits in Section II. Time-reversal of signals is carried out internal to the electromagnetic solvers, i.e., ideally.

2) Practical time-reversal. In this case, the FDTD and MoM solvers are coupled with the circuit simulator in Section III-A. To be specific, the electromagnetic solvers provide input signals to the ADS simulator and receive time-reversed signals from the circuit solver. In all the results in this section, $T_{0}=12.5$ ns, $\chi=5, N=18, \omega_{1}=2 \pi \times 3.2 \times 10^{9} \mathrm{rad} / \mathrm{s}$, and there is no noise present.

Two specific problem configurations are studied in this section. They are to simulate wireless communication and radar detection applications, respectively.
The wireless communication example is depicted in Fig. 16. Communication occurs in between a base station and three users. The base station is composed of 13 antenna elements; and the elements are geometrically arranged as in Fig. 16. There is one antenna at each user. All the antennas are assumed to be electrically small and behave as $z$-direction-oriented Hertzian dipoles with omni-directional radiation pattern in the $x-y$-plane. The three users are located at $(1.65 \mathrm{~m}, 1.65 \mathrm{~m}, 0)$, $(1.125 \mathrm{~m}, 1.65 \mathrm{~m}, 0)$, and $(1.65 \mathrm{~m}, 0.95 \mathrm{~m}, 0)$, respectively. The antenna element at the corner in the base station has coordinates $(0.15 \mathrm{~m}, 0.15 \mathrm{~m}, 0)$; and the distance among elements is 0.06 $\mathrm{m}$. A corner reflector made of two perfectly conducting plates is placed around the three users to make the problem configuration complicated. The two plates both have length $1.5 \mathrm{~m}$ and height $0.01 \mathrm{~m}$. Tip of the corner reflector sits at $(1.8 \mathrm{~m}, 1.8 \mathrm{~m}$, $0)$. It is not an easy task to achieve space division multiple access in the environment in Fig. 16. Suppose a regular phased array is implemented at the base station. Even if the phased array is able to deliver a narrow beam to a specific user, that beam would be bounced by the conducting plates and reach other users. The intention of this example is to demonstrate that space division multiple access could be accomplished through time-reversal together with the circuit system proposed in Section II. To establish a wireless communication link between User 1 and base station, User 1 transmits periodic short impulse by a current source excitation

$$
I_{z}(t)=e^{-\frac{\left(t-t_{\alpha}\right)^{2}}{2 \sigma^{2}}} \cos \left(\omega_{c} t\right)(\mathrm{A}), \quad \text { when } t \in\left[0, T_{0}\right]
$$




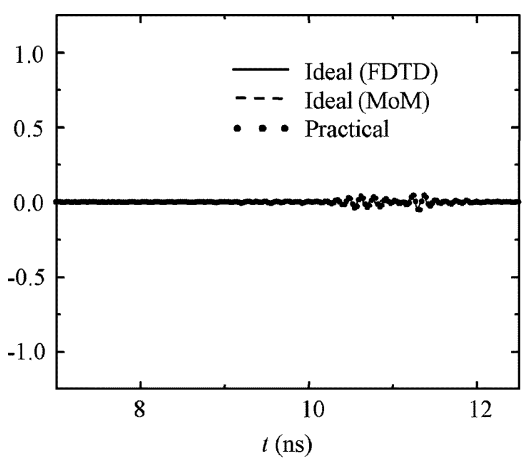

(a)

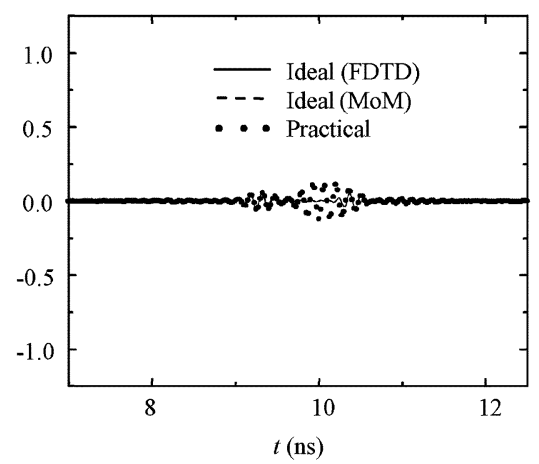

(b)

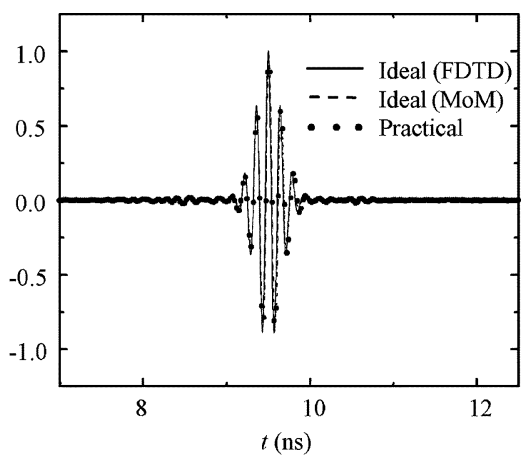

(c)

Fig. 20. Signals received at the three users when there is a link between the base station and User 3. (The vertical axes are normalized $E_{z}$.) (a) At User 1. (b) At User 2. (c) At User 3.

where $\sigma=138 \times 10^{-12} \mathrm{~s}, t_{\alpha}=0.9996 \times 10^{-9} \mathrm{~s}$, and $\omega_{c}=$ $2 \pi \times 6.5 \times 10^{9} \mathrm{rad} / \mathrm{s}$. (The signal in (15) virtually has spectral coverage of 3-10 GHz.) The fields radiated by User 1 are collected by all the 13 elements in the base station. Next, all the 13 elements time reverse their received signals and re-radiate them. Signals after ideal time-reversal and practical time-reversal are compared with one another in Fig. 17, where only one impulse out of the impulse train at the corner antenna element is plotted. Basically, ideal time-reversal and practical time-reversal match each other. As will be shown next, the small difference between them is not significant in the context of this communication example. When the radiations from the 13 elements of base station arrive at the three users, they are constructive at User 1, but destructive at the other two users. The signals received at the three users (electrical field along the $z$-direction and normalized by the maximum strength among the three users) are plotted in Fig. 18. Three sets of data are compared:

1) Results from FDTD solver with ideal time-reversal.

2) Results from MoM solver with ideal time-reversal.

3) Results from FDTD solver with practical time-reversal. The three sets of data have nice agreement, and they all show that the signal is strong at User 1, but weak at the other two users. It means that, a communication link between the base station and User 1 is established, with User 2 and User 3 excluded. Similar results are shown in Figs. 19 and 20 when the base station communicates with User 2 and User 3, respectively, both of which demonstrate space division multiple access phenomena. That is, when there is a link between base station and User 2, the other two users receive little signal; and similar phenomenon holds true for User 3. In Figs. 18-20, results from ideal time-reversal and practical time-reversal always match each other very well. Therefore, it is concluded that the time-reversal system proposed in Section II could realize space division multiple access in practical time-reversal wireless communication.

The last example in this section is related to iterative time-reversal radar detection [4]. The problem geometry is given in Fig. 21. In the free space, there is one radar and two targets. Both targets are of cubic shape and made of a perfect conductor. The cube on the left has side length $0.03 \mathrm{~m}$ and its center is located at $(0.135 \mathrm{~m}, 0.915 \mathrm{~m}, 0)$; the cube on the right is larger (side length $0.045 \mathrm{~m})$ and its center is at $(0.4575 \mathrm{~m}, 0.9225 \mathrm{~m}, 0)$. The radar consists of 13 antenna elements. As in the previous example,

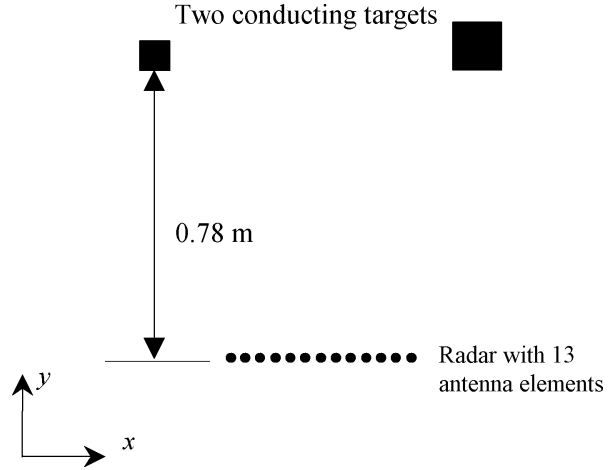

Fig. 21. Geometry of the radar detection example.

each antenna behaves as a $z$-direction oriented Hertzian dipole. The position of the left-most element is $(0.21 \mathrm{~m}, 0.12 \mathrm{~m}, 0)$; and distance among elements is $0.015 \mathrm{~m}$. To start the radar detection, the center element transmits periodic impulses to illuminate the two targets. The transmission is excited by a current source as in (15). The fields scattered by the two targets are then received by all 13 elements in the radar. Time-reversal is carried out at all the elements; and the reversed signals are radiated by all the antennas elements. The radiations from the 13 elements are focused onto the two targets. Since the two targets have different sizes, stronger fields are expected at the larger target. The above process could be repeated recursively. That is, the fields are scattered by the two targets; the scattered signals are received by the radar; the received fields are time-reversed and radiated to illuminate the targets again. According to [4], the contrast between the two targets would get larger and larger with more and more iterations. Such a phenomenon is also observed here. In Fig. 22, field strength along $y=0.9 \mathrm{~m}$ is plotted after the first, second, and third iterations. Specifically, field strength is represented by the maximum $\left|E_{z}\right|$ in the time history at a certain location. Also in Fig. 22, the field strength is normalized by the maximum value among all the locations along $y=0.9 \mathrm{~m}$. As in the previous example, three sets of data are compared with one another.

1) Results from FDTD solver with ideal time-reversal.

2) Results from MoM solver with ideal time-reversal.

3) Results from FDTD solver with practical time-reversal. 


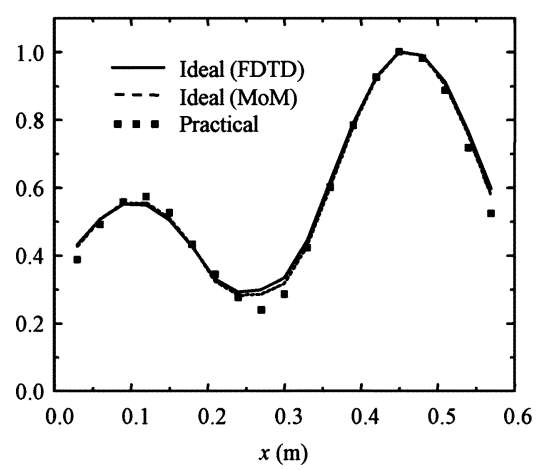

(a)

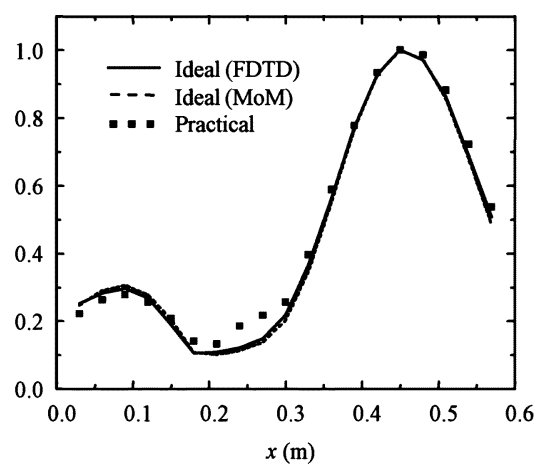

(b)

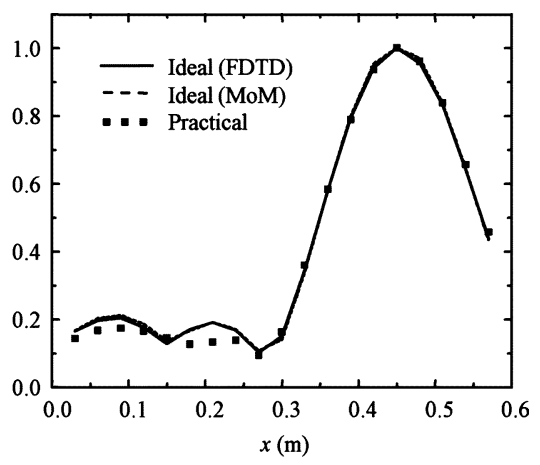

(c)

Fig. 22. Radar detection results at $y=0.9 \mathrm{~m}$. (Vertical axes are normalized maximum $\left|E_{z}\right|$.) (a) After the first iteration. (b) After the second iteration. (c) After the third iteration.

The three sets of data agree with one another, and they all clearly show the iterative focusing phenomena: after the first iteration, there are two focusing points; and, with more and more iterations, the left focal point gets weaker and weaker. It means that the time-reversal circuit system proposed in this paper can be applied to practical radar scenarios.

\section{CONCLUSION}

In this paper, a low-cost electronic circuit system is designed to time reverse short UWB impulses. The proposed system obtains discrete spectra of input impulses first; then achieves time-reversal in the frequency domain; and finally synthesizes time-reversed impulses using discrete continuous wave elements. This architecture is composed of commercially available semiconductor circuits, and hence, embodies a system-on-chip implementation of real-time time-reversal. Circuit-electromagnetic co-simulations using ADS and full-wave Maxwell's equations solvers demonstrate that the proposed system reliably accomplishes time-reversal of UWB impulses at the $3-10-\mathrm{GHz}$ frequency band in the context of realistic electromagnetic wave propagation environments. On-board prototyping of the proposed system is currently ongoing. A major limitation of our present system is that the number of local oscillators is strictly dictated by the temporal duration of impulses. Schemes of using frequency sweeping to relieve this limitation are under investigation.

\section{ACKNOWLEDGMENT}

The authors would like to acknowledge the Texas Advanced Computing Center (TACC), Arlington, TX, for granting access to its computational facilities.

\section{REFERENCES}

[1] A. Fannjiang and K. Solna, "Superresolution and duality for time-reversal of waves in random media," Phys. Lett. A, vol. 342, pp. 22-29, May 2005.

[2] G. Lerosey, J. de Rosny, A. Tourin, and M. Fink, "Focusing beyond the diffraction limit with far-field time reversal," Science, vol. 315, pp. 1120-1122, Feb. 2007.

[3] J. de Rosny and M. Fink, "Focusing properties of near-field time reversal," Phys. Rev. A, Gen. Phys., vol. 76, 2007, Art. ID 065801.
[4] M. Fink, "Time-reversal of ultrasonic fields-Part I: Basic principles," IEEE Trans. Ultrason., Ferroelectr., Freq. Control, vol. 39, no. 5, pp. 555-566, Sep. 1992.

[5] F. Wu, J.-L. Thomas, and M. Fink, "Time reversal of ultrasonic fields-Part II: Experimental results," IEEE Trans. Ultrason., Ferroelectr., Freq. Control, vol. 39, no. 5, pp. 567-578, Sep. 1992.

[6] M. Fink, "Time reversed acoustics," Phys. Today, vol. 50, pp. 34-40, Mar. 1997.

[7] M. Fink and C. Prada, "Acoustic time-reversal mirrors," Inverse Problems, vol. 17, no. 1, pp. R1-R38, 2001.

[8] H. C. Song, W. S. Hodgkiss, W. A. Kuperman, W. J. Higley, K. Raghukumar, and T. Akal, "Spatial diversity in passive time reversal communications," J. Acoust. Soc. Amer., vol. 120, no. 4, pp. 2067-2076, Oct. 2006

[9] S. G. Conti, P. Roux, and W. A. Kuperman, "Near-field time-reversal amplification," J. Acoust. Soc. Amer., vol. 121, no. 6, pp. 3602-3606, Jun. 2007.

[10] G. F. Edelmann, T. Akal, W. S. Hodgkiss, S. Kim, W. A. Kuperman, and H. C. Song, "An initial demonstration of underwater acoustic communication using time reversal," IEEE J. Ocean. Eng., vol. 27, no. 3, pp. 602-609, Jul. 2002.

[11] L. Borcea, G. Papanicolaou, C. Tsogka, and J. Berryman, "Imaging and time-reversal in random media," Inverse Problems, vol. 18, pp. 1247-1279, 2002.

[12] P. Blomberg, G. Papanicolaou, and H. K. Zhao, "Super-resolution in time-reversal acoustics," J. Acoust. Soc. Amer., vol. 111, pp. 230-248, 2002.

[13] E. Kerbrat, C. Prada, and D. Cassereau, "Ultrasonic nondestructive testing of scattering media using the decomposition of the time-reversal operator," IEEE Trans. Ultrason., Ferroelectr., Freq. Control, vol. 49, no. 8, pp. 1103-1112, Aug. 2002.

[14] R. Sorrentino, L. Roselli, and P. Mezzanotte, "Time reversal in finite difference time domain method," IEEE Microw. Guided Wave Lett., vol. 3, no. 11, pp. 402-404, Nov. 1993.

[15] M. Forest and W. J. R. Hoefer, "A novel synthesis technique for conducting scatterers using TLM time reversal," IEEE Trans. Microw. Theory Tech., vol. 43, no. 6, pp. 1371-1378, Jun. 1995.

[16] Y. Chang, H. R. Fetterman, I. L. Newberg, and S. K. Panaretos, "Microwave phase conjugation using antenna arrays," IEEE Trans. Microw. Theory Tech., vol. 46, no. 11, pp. 1910-1919, Nov. 1998.

[17] Y.-H. Pan, T.-Y. Huang, and R.-B. Wu, "Steady-state response by timereversal FDTD method with Lanczos algorithm," presented at the 2003 IEEE Int. AP-S Symp., Columbus, OH, Jun. 2003.

[18] B. E. Henty and D. D. Stancil, "Multipath-enabled super-resolution for RF and microwave communication using phase-conjugate arrays," Phys. Rev. Lett., vol. 93, Dec. 2004, Art ID 243904.

[19] C. Oestges, A. D. Kim, G. Papanicolaou, and A. J. Paulraj, "Characterization of space-time focusing in time-reversed random fields," IEEE Trans. Antennas Propag., vol. 53, no. 1, pp. 283-293, Jan. 2005.

[20] D. H. Chambers and J. G. Berryman, "Analysis of the time-reversal operator for a small spherical scatterer in an eletromagnetic field," IEEE Trans. Antennas Propag., vol. 52, no. 7, pp. 1729-1738, Jul. 2004. 
[21] L. Chiu, T. Y. Yum, W. S. Chang, Q. Xue, and C. H. Chan, "Retrodirective array for RFID and microwave tracking beacon applications," Microw. Opt. Technol. Lett., vol. 48, no. 2, pp. 409-411, Feb. 2006.

[22] G. Micolau, M. Saillard, and P. Borderies, "DORT method as applied to ultrawideband signals for detection of buried objects," IEEE Trans. Geosci. Remote Sens., vol. 41, no. 8, pp. 1813-1820, Aug. 2003.

[23] R. Carminati, R. Pierrat, J. De Rosny, and M. Fink, "Theory of the time reversal cavity for electromagnetic field," Opt. Lett., vol. 32, no. 21, pp. 3107-3109, Nov. 2007.

[24] M. E. Yavuz and F. L. Teixeira, "Space-frequency ultrawideband timereversal imaging," IEEE Trans. Geosci. Remote Sens., vol. 46, no. 4, pp. 1115-1124, Apr. 2008.

[25] J. C. Adams, W. Gregorwich, L. Capots, and D. Liccaardo, "Ultrawideband for navigation and communications," presented at the 2001 IEEE Aerosp. Conf., Big Sky, MT, Mar. 2001.

[26] G. R. Aiello, "Challenges for ultra-wideband (UWB) CMOS integration," presented at the 2003 IEEE MTT-S Int. Microw. Symp., Philadelphia, PA, Jun. 2003.

[27] D. Porcino and W. Hirt, "Ultra-wideband radio technology: Potential and challenges ahead," IEEE Commun. Mag., vol. 41, pp. 66-74, 2003.

[28] P. Kyritsi, G. Papnicolaou, P. Eggers, and A. Oprea, "One-bit time reversal for WLAN applications," presented at the IEEE 16th Int. Pers., Indoor, Mobile Radio Commun. Symp., Berlin, Germany, Sep. 2005.

[29] A. E. Akogua, R. C. Qiu, and N. Guo, "Demonstrating time reversal in ultra-wideband communications using time domain measurements," presented at the 51st IEEE Int. Instrum. Symp., Knoxville, TN, May 2005.

[30] C. Oestges, J. Hansen, S. M. Emami, A. D. Kim, G. Papanicalaou, and A. J. Paulraj, "Time reversal techniques for broad band wireless communication systems," presented at the 34th Eur. Microw. Conf., Amsterdam, The Netherlands, Oct. 2004.

[31] H. T. Nguyen, J. B. Anderson, and G. F. Pedersen, "The potential use of time reversal technique in multiple elements antenna systems," IEEE Commun. Lett., vol. 9, no. 1, pp. 40-42, Jan. 2005.

[32] R. C. Qiu, "A theory of time-reversed impulse multiple-input multiple-output (MIMO) for ultra-wideband (UWB) communications," presented at the IEEE Int. Ultra-Wideband Conf., Singapore, Sep. 2007.

[33] X. Liu, B. Z. Wang, S. Xiao, and J. Deng, "Performance of impulse radio UWB communications based on time reversal technique," Progr. Electromagn. Res., vol. PIER 79, pp. 401-413, 2008.

[34] R. C. Qiu, C. J. Zhou, J. Q. Zhang, and N. Guo, "Channel reciprocity and time-reversed propagation for ultra-wideband communications," presented at the 2007 IEEE Int. Antennas Propag. Symp., Honolulu, HI, Jun. 2007.

[35] D. Abbasi-Moghadam and V. T. Vakili, "Time reversal technique for ultra wideband (TR-UWB) communication systems and its performance analysis," presented at the Int. Telecommun. Symp., Tehran, Iran, Aug. 2008

[36] A. C. Fannjiang, "Time reversal communication in Rayleigh-fading broadcast channels with pinholes," Phys. Lett. A, vol. 353, pp. 389-397, 2006.

[37] S. Q. Xiao, J. Chen, B. Z. Wang, and X. F. Liu, "A numerical study on time-reversal electromagnetic wave for indoor ultra-wideband signal transmission," Progr. Electromagn. Res., vol. 77, pp. 329-342, 2007.

[38] Z. Yun and M. F. Iskander, "Time reversal with single antenna systems in indoor multipath environments: Spatial focusing and time compression," presented at the IEEE Int. Antennas Propag. Symp., Albuquerque, NM, Jul. 2006.

[39] K. Sarabandi, I. Koh, and M. D. Casciato, "Demonstration of time reversal methods in a multi-path environment," presented at the IEEE Int. Antennas Propag. Symp., Monterey, CA, Jun. 2004.

[40] E. Slob and K. Wapenaar, "GPR without a source: Cross-correlation and cross-convolution methods," IEEE Trans. Geosci. Remote Sens., vol. 45, no. 8, pp. 2501-2510, Aug. 2007.

[41] P. Kosmas and C. M. Rappaport, "Time reversal with the FDTD method for microwave breast cancer detection," IEEE Trans. Microw. Theory Tech., vol. 53, no. 7, pp. 2317-2323, Jul. 2005.

[42] P. Kosmas and C. M. Rappaport, "A matched-filter FDTD-based time reversal approach for microwave breast cancer detection," IEEE Trans. Antennas Propag., vol. 54, no. 4, pp. 1257-1264, Apr. 2006.

[43] L. Carin, D. Liu, W. Lin, and B. Guo, "Compressive sensing for multi-static scattering analysis," J. Comput. Phys., vol. 228, no. 9, pp. 3464-3477, May 2009.
[44] D. Singh, Z. Hu, and R. Qiu, "UWB channel sounding and channel characteristics in rectangular metal cavity," presented at the IEEE Southeastcon, Huntsville, AL, Apr. 2008.

[45] G. Lerosey, J. de Rosny, A. Tourin, A. Derode, G. Montaldo, and M Fink, "Time reversal of electromagnetic waves," Phys. Rev. Lett., vol. 92, no. 19, May 2004, Art. ID 193904

[46] G. Lerosey, J. de Rosny, A. Tourin, A. Derode, and M. Fink, "Time reversal of wideband microwaves," Appl. Phys. Lett., vol. 88, 2006, Art. ID 154101

[47] X. Zhou, P. Kyritsi, J. Martinez, A. Adenet, C. Lemasson, and P. C. F. Eggers, "Assessment of MISO time reversal for short-range communications in the $5 \mathrm{GHz}$ ISM band," Wireless Pers. Commun., vol. 43, pp. 759-776, 2007.

[48] P. Kyritsi, G. Papanicolaou, P. Eggers, and A. Oprea, "MISO time reversal and delay-spread compression for FWA channels at 5 GHz," IEEE Antenna Wireless Propag. Lett., vol. 3, no. 1, pp. 96-99, 2004.

[49] G. Lerosey, J. de Rosny, A. Tourin, A. Derode, G. Montaldo, and M. Fink, "Time reversal of electromagnetic waves and telecommunication," Radio Sci., vol. 40, no. 6, Sep. 2005, Art. ID RS6S12.

[50] H. T. Nguyen, J. B. Andersen, G. F. Pedersen, P. Kyritsi, and P. C. F. Eggers, "Time reversal in wireless communications: A measurementbased investigation," IEEE Trans. Wireless Commun., vol. 5, no. 8, pp. 2242-2252, Aug. 2006

[51] R. C. Qiu, C. J. Zhou, N. Guo, and J. Q. Zhang, "Time reversal with MISO for ultrawideband communications: Experimental results," IEEE Antennas Wireless Propag. Lett., vol. 5, no. 1, pp. 269-273, Dec. 2006.

[52] S. M. Anlage, J. Rodgers, S. Hemmady, J. Hart, T. M. Antonsen, and E. Ott, "New results in chaotic time-reversed electromagnetics: High frequency one-recording-channel time-reversal mirror," Acta Phys. Pol. A, vol. 112, no. 4, pp. 569-574, May 2007.

[53] D. Liu, S. Vasudevan, J. Krolik, G. Bal, and L. Carin, "Electromagnetic time-reversal source localization in changing media: Experiment and analysis," IEEE Trans. Antennas Propag., vol. 55, no. 2, pp. 344-354, Feb. 2007.

[54] D. Liu, G. Kang, L. Li, Y. Chen, S. Vasudevan, W. Joines, Q. H. Liu, J. Krolik, and L. Carin, "Electromagnetic time-reversal imaging of a target in a cluttered environment," IEEE Trans. Antennas Propag., vol. 53, no. 9, pp. 3058-3066, Sep. 2005.

[55] D. Liu, J. Krolik, and L. Carin, "Electromagnetic target detection in uncertain media: Time-reversal and minimum-variance algorithms," IEEE Trans. Geosci. Remote Sens., vol. 45, no. 4, pp. 934-944, Apr. 2007.

[56] G. Bal, L. Carin, D. Liu, and K. Ren, "Experimental validation of a transport-based imaging method in highly scattering environments," Inverse Problems, vol. 23, pp. 2527-2539, 2007

[57] J. M. F. Moura and Y. Jin, "Detection by time reversal: Single antenna," IEEE Trans. Signal Process., vol. 55, no. 1, pp. 187-201, Jan. 2007.

[58] J. M. F. Moura and Y. Jin, "Time reversal imaging by adaptive interference canceling," IEEE Trans. Signal Process., vol. 56, no. 1, pp. 233-247, Jan. 2008.

[59] F. Coppinger, A. S. Bhushan, and B. Jalali, "Time reversal of broadband microwave signals," Electron. Lett., vol. 35, no. 15, pp. 1230-1232, 1999.

[60] S. Hoyos and B. M. Sadler, "UWB mixed-signal transform-domain direct-sequence receiver," IEEE Trans. Wireless Commun., vol. 6, no. 8, pp. 3038-3046, Aug. 2007

[61] A. Medi and W. Namgoong, "A high data-rate energy-efficient interference-tolerant fully integrated CMOS frequency channelized UWB transceiver for impulse radio," IEEE J. Solid-State Circuits, vol. 43, no. 4, pp. 974-980, Apr. 2008.

[62] R. Thirugnanam and D. S. Ha, "A feasibility study on frequency domain ADC for impulse-UWB receivers," presented at the 4th Int. Circuits Syst. Commun. Conf., Shanghai, China, 2008.

[63] G. S. Gill, "Ultra-wideband radar using Fourier synthesized waveforms," IEEE Trans. Electromagn. Compat., vol. 39, no. 2, pp. 124-131, May 1997.

[64] V. Shenoy, S. Sha, S. Jung, and M. Lu, "A circuit implementation for time-reversal of short impulses," presented at the Asia-Pacific Microw. Conf., Hong Kong, China, Dec. 2008.

[65] A. Taflove and S. C. Hagness, Computational Electrodynamics: The Finite-Difference Time-Domain Method, 3rd ed. Norwood, MA: Artech House, 2005.

[66] R. F. Harrington, Field Computation by Moment Methods. New York: Macmillan, 1968 


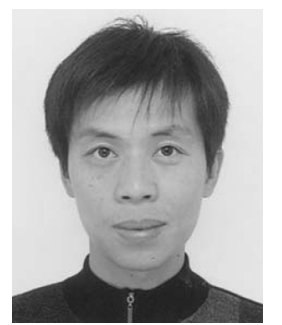

Huiqing Zhai was born in Jilin Province, China. He received the B.S., M.S., and Ph.D. degrees in electromagnetic fields and microwave technology from Xidian University, Xi' an, China, in 2000, 2003, and 2004, respectively.

In 2004, he joined the School of Electrical Engineering, Xidian University. From April 2005 to March 2008, he was with Tohoku University, Sendai, Japan, as a Research Fellow. He is currently a Postdoctoral Research Associate with The University of Texas at Arlington. His primary research interests include computational electromagnetics, microwave remote sensing, and microwave circuits and antennas for wireless communication.

Dr. Zhai was the recipeint of the Japan Society for Promotion of Science (JSPS) Research Fellowship from April 2006 to March 2008. He was also the recipient of the Best Paper Award and Zen'iti Kiyasu Award presented by the Institute of Electronics, Information and Communication Engineers (IEICE), Japan, 2008.

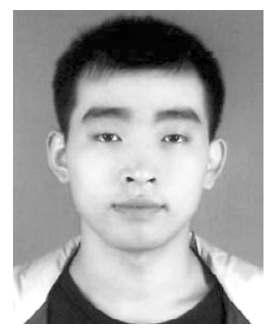

Shaoshu Sha was born in Xuzhou, China, in 1984. $\mathrm{He}$ received the Bachelor of Science degree in information engineering from Southeast University, Nanjing, China, in 2003, and the Master of Science degree in electrical engineering from The University of Texas at Arlington, in 2009.

From 2007 to 2009, he was a Graduate Research Assistant with the Department of Electrical Engineering in the University of Texas at Arlington. His research interests include antenna design, computational electromagnetics, and time-reversal electronic

system design.

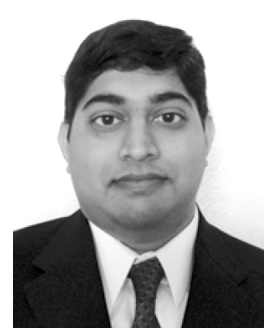

Varun K. Shenoy (S'08) received the B.E. degree in electrical engineering from the Manipal Institute of Technology, Manipal, Karnataka, India, in 205, the M.S. degree in electrical engineering from The University of Texas at Arlington, in 2007, and is currently working toward the Ph.D. degree at The University of Texas at Arlington.

His research interests include high-speed analog/RF CMOS circuits, UWB transceiver designs, and radar system designs.

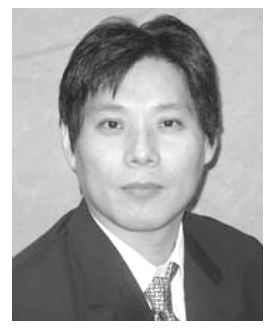

Sungyong Jung (M'02-SM'08) received the B.S. and M.S. degrees in electronics engineering from Yeungnam University, Kyeongsan, Korea, in 1991 and in 1993, respectively, and the Ph.D. degree in electrical engineering from the Georgia Institute of Technology, Atlanta, in 2002.

From 2001 to 2002, he was an Advanced Circuit Engineer with Quellan Inc., Atlanta, GA. He is currently an Associate Professor with the Department of Electrical Engineering, The University of Texas at Arlington. His research interests include UWB wireless integrated circuit (IC) and system design for wireless communications and radar applications, high-speed CMOS analog and mixed-signal circuit design, opto-electronic IC design, and modeling of high-speed circuit parasitics.

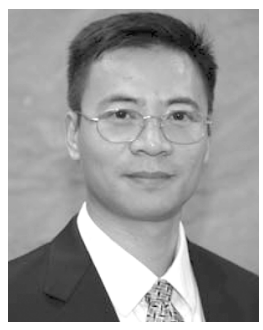

Mingyu Lu (M'03-SM'08) received the B.S. and M.S. degrees in electrical engineering from Tsinghua University, Beijing, China, in 1995 and 1997 respectively, and the Ph.D. degree in electrical engineering from the University of Illinois at Urbana-Champaign, in 2002.

From 1997 to 2002, he was a Research Assistant with the Department of Electrical and Computer Engineering, University of Illinois at Urbana-Champaign. From 2002 to 2005, he was a Postdoctoral Research Associate with the Electromagnetics
Laboratory, University of Illinois at Urbana-Champaign. In 2005, he joined the faculty of the Department of Electrical Engineering, The University of Texas at Arlington, as an Assistant Professor. His current research interests include radar systems, antenna design, computational electromagnetics, and microwave remote sensing.

Dr. Lu was the recipient of the First Prize Award in the Student Paper Competition of the 2001 IEEE Antennas and Propagation (IEEE AP-S) International Symposium, Boston, MA. He was also the recipient of the 2008 Outstanding Service Award presented by the IEEE Fort Worth Chapter.

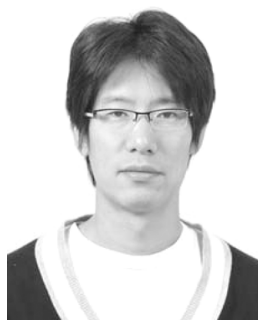

Kyoungwon Min was born in Seoul, Korea, in 1971. He received the B.S. and M.S. degrees in electronics engineering from the Hongik University, Seoul, Korea, in 1993 and 1995, respectively, and is currently working toward the Ph.D. degree in electronics engineering at Yonsei University, Seoul, Korea.

In 1995, he joined the Korea Electronics Technology Institute, Gyeonggi-Do, Korea. $\mathrm{He}$ is currently the Managerial Researcher of hybrid signal processing with the System-on-Chip (SoC) Center, Korea Electronics Technology Institute. His research interests include very large scale integration (VLSI) and SoC design for sensor and image signal processing.

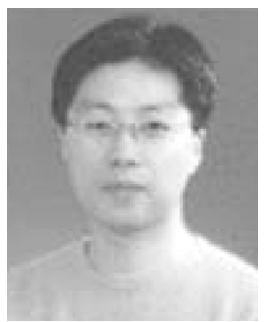

Sungchul Lee was born in Jeonju, Korea, in 1971. $\mathrm{He}$ received the B.S. and M.S. degrees from the School of Information and Telecommunication Engineering, Chonbuk National University, Jeonju, Korea, in 1993 and 1995, respectively, from, and the Ph.D. degree from the School of Electronics Engineering, Chonbuk National University, Jeonju, Korea, in 2008 .

Since 1995, he has been with the Korea Electronics Technology Institute, Gyeonggi-Do, Korea, where he has been involved with research and development with the System IC Research and Development Division, where he currently is a Managerial Researcher and Team Leader. His research interests are in the areas of high-speed I/O, RF/analog IC design, and sensor signal processing.

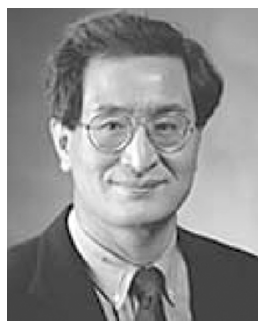

Dong S. Ha (M'86-SM'97-F'08) received the B.S. degree in electrical engineering from Seoul National University, Seoul, Korea, in 1974, and the M.S. and $\mathrm{Ph} . \mathrm{D}$. degrees in electrical and computer engineering from the University of Iowa, Iowa City, in 1984 and 1986, respectively.

Since Fall 1986, he has been a faculty member with the Department of Electrical and Computer Engineering, Virginia Polytechnic Institute of and State University, Blacksburg. He is currently a Professor and Director of the Center for Embedded Systems and Critical Applications (CESCA). He supervises the VLSI for Telecommunications (VTVT) Group, Virginia Polytechnic Institute of and State University, which specializes in low-power VLSI design for various applications including wireless communications. Along with his students, he has developed four computer-aided design tools for digital circuit testing and CMOS standard cell libraries. The library cells and the source code for the four tools have been distributed to about 300 universities and research institutions worldwide. His research interests include structural health monitoring systems, energy harvesting circuits, wireless body area networks, power-line communications for self-contained systems, low-power analog and mixed-signal design, and RF IC design.

Dr. Ha was general chair of the 2005 System-on-Chip Conference (SOCC) and Technical Program chair of the 2003 and 2004 SOCC. 